PROCEEDINGS OF THE

AMERICAN MATHEMATICAL SOCIETY

Volume 135, Number 2, February 2007, Pages 461-467

S 0002-9939(06)08497-8

Article electronically published on September 11, 2006

\title{
SPECTRUM OF A COMPACT WEIGHTED COMPOSITION OPERATOR
}

\author{
GAJATH GUNATILLAKE
}

(Communicated by Joseph A. Ball)

\begin{abstract}
For $\psi$ analytic in the open unit disk and $\varphi$ an analytic map from the unit disk into itself, the weighted composition operator $C_{\psi, \varphi}$ is the operator on the weighted Hardy space $H^{2}(\beta)$ given by $\left(C_{\psi, \varphi} f\right)(z)=\psi(z) f(\varphi(z))$. This paper discusses the spectrum of $C_{\psi, \varphi}$ when it is compact on a certain class of weighted Hardy spaces and when the composition $\operatorname{map} \varphi$ has a fixed point inside the open unit disk.
\end{abstract}

\section{INTRODUCTION}

A weighted composition operator $C_{\psi, \varphi}$ maps an analytic function $f$ on the unit disk $D$ into $\left(C_{\psi, \varphi} f\right)(z)=\psi(z) f(\varphi(z))$. These operators come up naturally. For example, Forelli showed that an isometry on $H^{p}$ for $1<p<\infty$ and $p \neq 2$ is a weighted composition operator 4 . Though somewhat unrelated to the spaces discussed here, backward weighted shifts on sequence spaces are weighted composition operators, too.

To avoid $C_{\psi, \varphi}$ being merely a multiplication operator, the composition map $\varphi$ is taken to be different from identity. Theorem 11 which is the main result of this paper, asserts that if $C_{\psi, \varphi}$ is a compact operator on the weighted Hardy space $H^{2}(\beta)$ and if the Denjoy-Wolff point of $\varphi$ is inside the open unit disk, then the spectrum of $C_{\psi, \varphi}$ is the set $\left\{0, \psi(a), \psi(a) \varphi^{\prime}(a), \psi(a)\left(\varphi^{\prime}(a)\right)^{2}, \psi(a)\left(\varphi^{\prime}(a)\right)^{3}, \cdots\right\}$, where $a$ is the Denjoy-Wolff point of $\varphi$. A similar result that applies only to the classical Hardy space has appeared in recent work of Shapiro and Smith [7, pages 76-77] and Clifford and Dabkowski [2, pages 186-187]. Also the doctoral dissertation of Hammond [6] contains a similar result obtained by using a different technique. In addition, the proofs of Lemmas 2 and 3 were inspired by material in [3, pages 270-275], and the proof of Theorem 2 was inspired by the proof of Theorem 7.14 of [3].

The proof of Theorem 1 is divided into two parts. First we show that if $\lambda$ is an eigenvalue, then it must be of the form $\psi(a)\left(\varphi^{\prime}(a)\right)^{j}$ for some non-negative integer $j$.

Next we identify the actual eigenvalues among these numbers by finding eigenvalues of the adjoint operator $C_{\psi, \varphi}^{*}$. First we find a finite-dimensional invariant subspace of the adjoint. This allows us to represent the adjoint as a $2 \times 2$ upper

Received by the editors February 3, 2005 and, in revised form, September 19, 2005.

2000 Mathematics Subject Classification. Primary 47B32.

(C)2006 American Mathematical Society Reverts to public domain 28 years from publication 
triangular block matrix. Eigenvalues of $C_{\psi, \varphi}^{*}$ can be found by looking at this block matrix.

After finding the spectrum when the composition map has a fixed point inside the unit disk, we classify a class of compact weighted composition operators where the composition map $\varphi$ must have its Denjoy-Wolff point inside the open unit disk. Thus the spectrum for this class of operators can be computed using Theorem 1 .

Compact weighted composition operators on the Hardy space $H^{2}(D)$, where the composition map has fixed points on the unit circle, are discussed in 1]. We find the spectrum of such operators.

\section{Preliminaries}

Let $f$ be an analytic map on the open unit disk $D$ given by the Taylor series $f(z)=a_{0}+a_{1} z+a_{2} z^{2}+\cdots$ and let $\{\beta(j)\}_{j=0}^{\infty}$ be a sequence of positive numbers where $\liminf _{j \rightarrow \infty} \beta(j)^{1 / j} \geq 1$. Then $f$ is in the space $H^{2}(\beta)$ if and only if $\sum_{j=0}^{\infty}\left|a_{j}\right|^{2} \beta(j)^{2}<\infty$. If $f(z)=\sum_{j=0}^{\infty} a_{j} z^{j}$ and $g(z)=\sum_{j=0}^{\infty} b_{j} z^{j}$ are two functions in $H^{2}(\beta)$, an inner product can be defined by

$$
\langle f, g\rangle=\sum_{j=0}^{\infty} a_{j} \overline{b_{j}} \beta(j)^{2} .
$$

The space $H^{2}(\beta)$ becomes a Hilbert space with this inner product [3, page 14]. If $\beta(j)=1$ for all $j$ the space $H^{2}(\beta)$ is known as the Hardy Hilbert space, if $\beta(j)^{2}=1 /(j+1)$ it is known as the Bergman Hilbert space, and if $\beta(j)^{2}=j+1$ it is the Dirichlet Hilbert space.

Let $w$ be a point on the open unit disk. Define

$$
K_{w}(z)=\sum_{j=0}^{\infty} \frac{z^{j} \bar{w}^{j}}{\beta(j)^{2}} .
$$

Then $K_{w}$ is in $H^{2}(\beta)$ and $\left\|K_{w}\right\|^{2}=\sum_{j=0}^{\infty}|w|^{2 j} / \beta(j)^{2}$ (see [3, page16]). Thus $\left\|K_{w}\right\|$ is an increasing function of $|w|$. If $f(z)=\sum_{j=0}^{\infty} a_{j} z^{j}$, then

$$
\left\langle f, K_{w}\right\rangle=\sum_{j=0}^{\infty} \frac{a_{j} w^{j} \beta(j)^{2}}{\beta(j)^{2}}=f(w) .
$$

Therefore

$$
\left\langle f, K_{w}\right\rangle=f(w)
$$

for all $f$, and $K_{w}$ is known as the point evaluation kernel at $w$. It can be easily shown that $C_{\psi, \varphi}^{*} K_{w}=\overline{\psi(w)} K_{\varphi(w)}$.

Let $\varphi$ be an analytic map from the open unit disk into itself and let $\zeta$ be a point on the unit circle. Then $\lim _{r \rightarrow 1^{-}} \varphi(r \zeta)$ exists for almost all $\zeta$ on the unit circle; by abusing the notation we will denote this limit by $\varphi(\zeta)$. If the limit is $\zeta$ we say $\zeta$ is a fixed point of $\varphi$ on the unit circle. Similarly if $\lim _{r \rightarrow 1^{-}} \varphi^{\prime}(r \zeta)$ exists for some $\zeta$ on the unit circle, then we will denote this limit by $\varphi^{\prime}(\zeta)$ [3, page 50].

Suppose $\varphi$ is a map from the unit disk into itself which is different from the identity. Then it can have at most one fixed point inside the open unit disk. If $\varphi$ has a fixed point in the open unit disk, the absolute value of the derivative of $\varphi$ at the fixed point will be less than or equal to 1 . If $\varphi$ does not have a fixed point in the open disk, there is at least one fixed point $\zeta$ on the unit circle and there is exactly one fixed point on the unit circle with $\left|\varphi^{\prime}(\zeta)\right| \leqslant 1$. When $\varphi$ is not an 
elliptic automorphism this distinguished fixed point, $a$, where $|a| \leq 1, \varphi(a)=a$, and $\varphi^{\prime}(a) \leq 1$, is known as the Denjoy-Wolff point of $\varphi$ (see [3, pages 50-59]).

In the lemma below we identify all possible eigenvalues of $C_{\psi, \varphi}$.

Lemma 1. Suppose $C_{\psi, \varphi}$ is a compact operator on the weighted Hardy space $H^{2}(\beta)$. If the Denjoy-Wolff point a of the composition map $\varphi$ is inside the open unit disk, then the set

$$
\left\{0, \psi(a), \psi(a) \varphi^{\prime}(a), \psi(a)\left(\varphi^{\prime}(a)\right)^{2}, \psi(a)\left(\varphi^{\prime}(a)\right)^{3}, \cdots\right\}
$$

contains the spectrum.

Proof. A compact operator on an infinite-dimensional space is not invertible, hence 0 is in the spectrum. Now we show that if $\lambda$ is an eigenvalue, then $\lambda$ must be of the form $\psi(a)\left(\varphi^{\prime}(a)\right)^{n}$. If $\lambda$ is a non-zero eigenvalue, then

$$
\psi(z) f(\varphi(z))=\lambda f(z)
$$

for some non-zero $f$ which is holomorphic on the unit disk. Let $f$ have a zero of order $n$ at $a$. If $n=0$ put $z=a$, and we get that $\lambda=\psi(a)$. For $n>0$ differentiate (11) $n$ times. Then,

$$
\sum_{j=0}^{n-1} \alpha_{j}(z) f^{(j)}(\varphi(z))+\psi(z) f^{(n)}(\varphi(z))\left(\varphi^{\prime}(z)\right)^{n}=\lambda f^{(n)}(z),
$$

where $f^{(k)}$ stands for the $k^{\text {th }}$ derivative of $f$ and $\alpha_{j}$ 's are functions which consist of various products of derivatives of $\psi$ and $\varphi$. The exact values of these are not important to us. Now let $z=a$. Since $f$ has a zero of order of $n$ at $a$, all the terms in the sum $\sum_{j=0}^{n-1}$ vanish, and this yields $\lambda=\psi(a)\left(\varphi^{\prime}(a)\right)^{n}$. The spectrum of a compact operator consists only of eigenvalues, and the above computation shows that only possible eigenvalues are of the form $\psi(a)\left(\varphi^{\prime}(a)\right)^{n}$. This completes the proof.

To find the spectrum we need to find which of the numbers discussed in Lemma 1 are really eigenvalues. To do that, we look at the adjoint of this operator and a special class of continuous linear functionals on $H^{2}(\beta)$.

Definition 1. Let $j$ be a positive integer. If

$$
K_{a}^{[j]}(z)=\frac{d^{j} K_{a}(z)}{d \bar{w}^{j}},
$$

then $K_{a}^{[j]}$ is in $H^{2}(\beta)$ and $\left\langle f, K_{a}^{[j]}\right\rangle=f^{(j)}(a)$ where $f^{(j)}(a)$ is the $j^{\text {th }}$ derivative of $f$ at $a$. The function $K_{a}^{[j]}$ is known as the $j^{t h}$ derivative evaluation kernel at $a$ (see [3, Theorem 2.16]).

Lemma 2. Let $m$ be a positive integer. Then the span of $\left\{K_{a}, K_{a}^{[1]}, \cdots, K_{a}^{[m]}\right\}$ is an invariant subspace of $C_{\psi, \varphi}^{*}$.

Proof. Let $f$ be any function on $H^{2}(\beta)$. Then

$$
\begin{aligned}
\left\langle f, C_{\psi, \varphi}^{*} K_{a}^{[n]}\right\rangle & =\left\langle C_{\psi, \varphi} f, K_{a}^{[n]}\right\rangle \\
& =\left\langle\psi f \circ \varphi, K_{a}^{[n]}\right\rangle .
\end{aligned}
$$


Now $\psi f \circ \varphi$ is differentiated $n$ times and evaluated at $a$. This results in

$$
\left\langle f, C_{\psi, \varphi}^{*} K_{a}^{[n]}\right\rangle=\sum_{j=0}^{n-1} \alpha_{j}(a) f^{(j)}(a)+\psi(a) f^{(n)}(a)\left(\varphi^{\prime}(a)\right)^{n} .
$$

Now (5) can be written as

$$
\left\langle f, C_{\psi, \varphi}^{*} K_{a}^{[n]}\right\rangle=\sum_{j=0}^{n-1} \alpha_{j}(a)\left\langle f, K_{a}^{[j]}\right\rangle+\psi(a)\left(\varphi^{\prime}(a)\right)^{n}\left\langle f, K_{a}^{[n]}\right\rangle .
$$

Now using the algebraic properties of the inner product on the right-hand side of (6) , we get

$$
\left\langle f, C_{\psi, \varphi}^{*} K_{a}^{[n]}\right\rangle=\left\langle f, \quad \sum_{j=0}^{n-1} \overline{\alpha_{j}(a)} K_{a}^{[j]}+\overline{\psi(a)\left(\varphi^{\prime}(a)\right)^{n}} K_{a}^{[n]}\right\rangle .
$$

Since $f$ was arbitrary, this proves that

$$
C_{\psi, \varphi}^{*} K_{a}^{[n]}=\sum_{j=0}^{n-1} \overline{\alpha_{j}(a)} K_{a}^{[j]}+\overline{\psi(a)\left(\varphi^{\prime}(a)\right)^{n}} K_{a}^{[n]} .
$$

Note that the order of the highest derivative on the right-hand side does not exceed $n$. This completes the proof.

Lemma 3. Suppose $C_{\psi, \varphi}$ is a bounded operator on $H^{2}(\beta)$, and the Denjoy-Wolff point a of $\varphi$ is inside the open unit disk. Then $\overline{\psi(a)(\varphi(a))^{j}}$ is in the spectrum of the adjoint operator $C_{\psi, \varphi}^{*}$ for any non-negative integer $j$.

Proof. First we use (8) to compute the matrix representation of $C_{\psi, \varphi}^{*}$ restricted to the subspace discussed in Lemma 2, Let $\mathbf{K}_{m}$ denote the span of $\left\{K_{a}, K_{a}^{[1]}, \cdots, K_{a}^{[m]}\right\}$. This spanning set is also linearly independent and hence is a basis. The matrix of the operator restricted to $\mathbf{K}_{m}$ with respect to this basis is

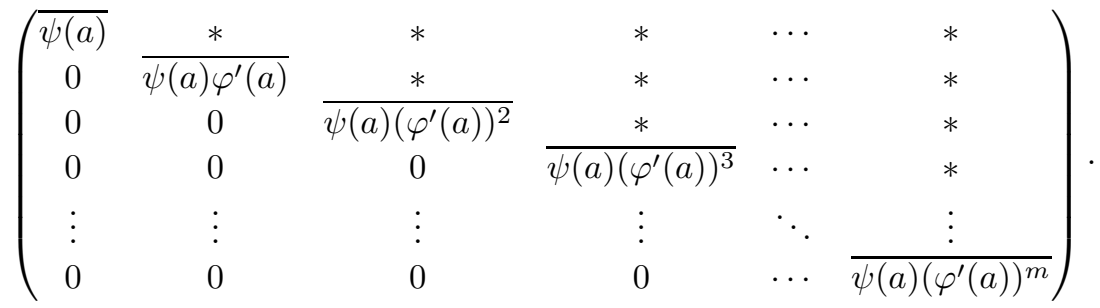

Let us call this matrix $A_{m}$. Then $A_{m}$ is an $(m+1) \times(m+1)$ upper triangular matrix. The ${ }^{*}$ 's denote $\overline{\alpha_{j}(a)}$ 's.

The subspace $\mathbf{K}_{m}$ is finite dimensional and therefore is closed. The Hardy space can be decomposed as $H^{2}(\beta)=\mathbf{K}_{m} \oplus \mathbf{K}_{m}^{\perp}$. The block matrix of $C_{\psi, \varphi}^{*}$ with respect to this decomposition is

$$
\left(\begin{array}{cc}
A_{m} & B \\
0 & C_{m}
\end{array}\right)
$$

The fact that $\mathbf{K}_{m}$ is invariant under $C_{\psi, \varphi}^{*}$ makes the lower left corner of this decomposition 0. Since there is a 0 at the lower left and the subspace $\mathbf{K}_{m}$ is finite dimensional, the spectrum of $C_{\psi, \varphi}^{*}$ is the union of the spectrum of $A_{m}$ and the spectrum of $C_{m}$ [3, page 270]. Since $A_{m}$ is a finite-dimensional upper triangular 
matrix, its spectrum consists of the diagonal values. Therefore we can conclude that the spectrum of $C_{\psi, \varphi}^{*}$ contains the set

$$
\left\{\overline{\psi(a)}, \overline{\psi(a)\left(\varphi^{\prime}(a)\right)}, \overline{\psi(a)\left(\varphi^{\prime}(a)\right)^{2}}, \cdots, \overline{\psi(a)\left(\varphi^{\prime}(a)\right)^{m}}\right\} .
$$

But $m$ is arbitrary, therefore we can see that any number of the form $\overline{\psi(a)(\varphi(a))^{j}}$, where $j$ is a non-negative integer, is in the spectrum of $C_{\psi, \varphi}^{*}$. This completes the proof.

Theorem 1. Let $C_{\psi, \varphi}$ be a compact operator on the weighted Hardy space $H^{2}(\beta)$. If the Denjoy-Wolff point of $\varphi$ is inside the disk, then the spectrum of $C_{\psi, \varphi}$ is the set

$$
\left\{0, \psi(a), \psi(a) \varphi^{\prime}(a), \psi(a)\left(\varphi^{\prime}(a)\right)^{2}, \psi(a)\left(\varphi^{\prime}(a)\right)^{3}, \cdots\right\},
$$

where $a$ is the Denjoy-Wolff point of $\varphi$.

Proof. If the Denjoy-Wolff point of $\varphi$ is $a$ and is inside the open disk, then for any positive integer $j$ the number $\psi(a)\left(\varphi^{\prime}(a)\right)^{j}$ is in the spectrum by Lemma 3. Clearly 0 is in the spectrum. Thus the spectrum contains the set

$$
\left\{0, \psi(a), \psi(a) \varphi^{\prime}(a), \psi(a)\left(\varphi^{\prime}(a)\right)^{2}, \psi(a)\left(\varphi^{\prime}(a)\right)^{3}, \cdots\right\} .
$$

By Lemma 1 this set contains the spectrum. This completes the proof.

Interestingly, it is possible for $C_{\psi, \varphi}$ to be compact even when $\psi$ is unbounded. For example if $\psi(z)=((1-z) /(1+z))^{1 / 4}$ and $\varphi(z)=(1+z) / 2$, then $C_{\psi, \varphi}$ is compact and also if $\psi(z)=(1+z)^{-1 / 4}$ and $\varphi(z)=z / 2$, then $C_{\psi, \varphi}$ is compact. This and other conditions for compactness are included in [5] and is the subject of a forthcoming paper by the author.

As we will see in the next theorem compactness of $C_{\psi, \varphi}$ for some weight functions $\psi$ force $\varphi$ to have a fixed point inside the disk.

Theorem 2. Let $C_{\psi, \varphi}$ be a compact operator on the space $H^{2}(\beta)$ where

$$
\sum 1 / \beta(j)^{2}=\infty
$$

If $\liminf _{r \rightarrow 1^{-}}|\psi(r \zeta)|>0$ for any $\zeta$ on the unit circle, then the composition map $\varphi$ will have its Denjoy-Wolff point inside the open unit disk.

Proof. By contradiction. Suppose $\varphi$ does not have a fixed point inside the disk. Then $\varphi$ must have its Denjoy-Wolff point on the unit circle [3, pages 50-59]. Let us call this point $\zeta$. Let $r$ belong to the interval $(0,1)$.

Now we apply the adjoint of $C_{\psi, \varphi}$ to the normalized kernel function $K_{r \zeta} /\left\|K_{r \zeta}\right\|$ :

$$
\left\|C_{\psi, \varphi}^{*} \frac{K_{r \zeta}}{\left\|K_{r \zeta}\right\|}\right\|=|\psi(r \zeta)| \frac{\left\|K_{\varphi(r \zeta)}\right\|}{\left\|K_{r \zeta}\right\|} .
$$

Since $\zeta$ is the Denjoy-Wolff point $|\varphi(r \zeta)| \geqslant|r \zeta|$ (see 3, page 49]). But $\| K_{w}||$ is an increasing function of $|w|$, therefore $\left\|K_{\varphi(r \zeta)}|| \geqslant\right\| K_{r \zeta} \|$. Now by using (10) we get

$$
\left\|C_{\psi, \varphi}^{*} \frac{K_{r \zeta}}{\left\|K_{r \zeta}\right\|}\right\| \geqslant|\psi(r \zeta)| .
$$

Normalized kernel functions $K_{r \zeta} /\left\|K_{r \zeta}\right\|$ converge weakly to zero as $r$ tends to 1 (see [3, Theorem 2.17]). Since $C_{\psi, \varphi}^{*}$ is compact, the left-hand side of (11) must tend to zero as $r$ tends 1 , but the right-hand side will be larger than some positive $\delta_{\zeta}$ by our hypothesis about $\psi$. This is a contradiction. Therefore $\varphi$ cannot have its 
Denjoy-Wolff point on the unit circle. Hence the Denjoy-Wolff point of $\varphi$ must be inside the open unit disk.

Theorem 2 and Theorem 1 give us the following corollary.

Corollary 1. Suppose $C_{\psi, \varphi}$ is a compact operator on $H^{2}(\beta)$ where $\sum 1 / \beta(j)^{2}=\infty$. If $\lim \inf _{r \rightarrow 1^{-}}|\psi(r \zeta)|>0$ for any $\zeta$ on the unit circle, then the spectrum of $C_{\psi, \varphi}$ is the set

$$
\left\{0, \psi(a), \psi(a) \varphi^{\prime}(a), \psi(a)\left(\varphi^{\prime}(a)\right)^{2}, \psi(a)\left(\varphi^{\prime}(a)\right)^{3}, \cdots\right\}
$$

where $a$ is the Denjoy-Wolff point of $\varphi$.

Lemma 3.3 of [1] gives a method to find $\psi$ so that $C_{\psi, \varphi}$ is compact on the Hardy space $H^{2}(D)$ when $\varphi$ has fixed points on the boundary. We discuss the spectrum for such operators. First we quote the lemma.

Lemma 4. Suppose $\psi$ is continuous on the closed unit disk with $\psi(1)=0$ and suppose further that the function $\theta \rightarrow \psi\left(e^{i \theta}\right)$ is differentiable at $\theta=0$. Then for every non-automorphic linear fractional $\varphi$ of the unit disk with fixed point at 1 , the operator $C_{\psi, \varphi}$ is compact on $H^{2}(D)$.

If $\varphi$ has a fixed point inside the disk in addition to the one on the unit circle, then Theorem 1 gives the spectrum. Therefore we compute the spectrum when $\varphi$ has no fixed points inside the open unit disk. We will denote the composition of $\varphi$ with itself $n$ times by $\varphi_{n}$. That is $\varphi_{n}=\varphi \circ \varphi \circ \cdots \circ \varphi$ ( $n$ times $)$.

Theorem 3. Suppose $\psi$ and $\varphi$ satisfy the hypothesis in Lemma 4, and $\varphi$ has no fixed points inside the open unit disk. Then $\sigma\left(C_{\psi, \varphi}\right)=\{0\}$.

Proof. We will show that the spectral radius of this operator is 0 . Since $\varphi$ is a non-automorphic linear fractional map with a fixed point at 1 , it takes the unit circle to a circle tangent to unit circle at 1 . The map $\varphi$ has no fixed points inside the open unit disk, so 1 is the Denjoy-Wolff point.

Let $\epsilon>0$. There is a $\delta>0$ such that $|\psi(z)|<\epsilon$ whenever $|z-1|<\delta$ and $z$ is in the closed unit disk. Let $W=\{z:|z-1|<\delta,|z| \leq 1\}$. Clearly $W$ is open in $\bar{D}$. Let $U=\varphi(D)$. Then $\bar{U}$ is tangent to the unit circle at 1 . Let $V=\overline{U-W}$; then $V$ is a compact subset of the open unit disk. Therefore the sequence $\left\{\varphi_{n}\right\}$ converges uniformly to 1 on $V$. Consider a point $e^{i \theta}$ on the unit circle. Then $\varphi\left(e^{i \theta}\right)$ is either in $W$ or $V$. If $\varphi\left(e^{i \theta}\right)$ is in $V$, then there is an $N$ that does not depend on $\theta$ such that $\varphi_{j}\left(e^{i \theta}\right)$ is in $W$ for all $j>N$. If $\varphi\left(e^{i \theta}\right)$ is not in $V$ consider the sequence $\left\{\varphi_{j}\left(e^{i \theta}\right)\right\}_{j=1}^{\infty}$; either $\varphi_{j}\left(e^{i \theta}\right)$ is in $W$ for all $j$ or $\varphi_{j}\left(e^{i \theta}\right)$ will be in $V$ for some $j$. If $\varphi_{j}\left(e^{i \theta}\right)$ is in $V$ for some $j$, take $j^{\prime}$ to be the smallest integer such that $\varphi_{j}\left(e^{i \theta}\right)$ is in $V$. Then $\varphi_{j}\left(e^{i \theta}\right)$ is in $W$ for all $j>j^{\prime}+N$. Therefore for any $e^{i \theta}$ on the unit circle at most $N$ terms of the sequence $\left\{\varphi_{j}\left(e^{i \theta}\right)\right\}_{j=1}^{\infty}$ will be outside $W$. Hence at most $N$ terms of the sequence $\left\{\left|\psi\left(\varphi_{j}\left(e^{i \theta}\right)\right)\right|\right\}_{j=1}^{\infty}$ will be larger than $\epsilon$ from the sequence for any $e^{i \theta}$. Also $\psi$ is bounded on the closed unit disk, therefore $\left|\psi\left(\varphi_{j}\left(e^{i \theta}\right)\right)\right|<M$ 
for some $M>0$. Now if $f$ is in $H^{2}(D)$ and $n>N$, then

$$
\begin{aligned}
\left\|C_{\psi, \varphi}^{n}(f)\right\|^{2} & =\int_{T}\left|\psi\left(e^{i \theta}\right)\right|^{2}\left|\psi\left(\varphi\left(e^{i \theta}\right)\right)\right|^{2} \cdots\left|\psi\left(\varphi_{n-1}\left(e^{i \theta}\right)\right)\right|^{2}\left|f\left(\varphi_{n}\left(e^{i \theta}\right)\right)\right|^{2} \frac{d \theta}{2 \pi} \\
& \leq \epsilon^{2(n-N-1)} M^{2(N+1)} \int_{T}\left|f\left(\varphi_{n}\left(e^{i \theta}\right)\right)\right|^{2} \frac{d \theta}{2 \pi} \\
& =\epsilon^{2(n-N-1)} M^{2(N+1)}\left\|C_{\varphi_{n}}(f)\right\|^{2} \\
& \leq \epsilon^{2(n-N-1)} M^{2(N+1)}\left\|C_{\varphi_{n}}\right\|^{2}\|(f)\|^{2},
\end{aligned}
$$

thus

$$
\left\|C_{\psi, \varphi}^{n}\right\| \leq \epsilon^{(n-N-1)} M^{(N+1)}\left\|C_{\varphi_{n}}\right\|,
$$

but $C_{\varphi_{n}}=C_{\varphi}^{n}$, therefore

$$
\left\|C_{\psi, \varphi}^{n}\right\| \leq \epsilon^{(n-N-1)} M^{(N+1)}\left\|C_{\varphi}^{n}\right\| .
$$

Hence for all $n$ large enough,

$$
\begin{aligned}
\left\|C_{\psi, \varphi}^{n}\right\|^{1 / n} & \leq \epsilon 2\left\|C_{\varphi}^{n}\right\|^{1 / n} \\
& \leq \epsilon 2\left\|C_{\varphi}\right\| .
\end{aligned}
$$

Thus we can see that the spectral radius of the operator is 0 . Therefore $\sigma\left(C_{\psi, \varphi}\right)=$ $\{0\}$. This completes the proof.

\section{ACKNOWLEDGEMENT}

The research for this paper was undertaken in partial fulfillment of the requirements for the author's Ph.D. at the Purdue University. The author thanks his thesis advisor Professor Carl Cowen for his guidance throughout the research.

\section{REFERENCES}

[1] P. Bourdan, D. Levi, S. Narayan, and J. Shapiro, Which linear-fractional composition operators are essentially normal?, JMAA 280(2003), 30-53. MR.1972190 (2003m:47042)

[2] J. Clifford and M. Dabkowski, Singular values and Schmidt pairs of composition operators on the Hardy space, JMAA 305(2005), 183-196. MR2128121 (2005m:47045)

[3] C. Cowen and B. MacCluer, Composition Operators on Spaces of Analytic Functions, CRC Press, Boca Raton, 1995. MR.1397026 (97i:47056)

[4] F. Forelli, The isometries of $H^{p}$, Canad. J. Math. 16(1964), 721-728. MR0169081 (29:6336)

[5] G. Gunatillake, Weighted Composition Operators, Doctoral Dissertation, Purdue University, 2005.

[6] C. Hammond, On the Norm of a Composition Operator, Doctoral Dissertation, University of Virginia, 2003.

[7] J. Shapiro and W. Smith, Hardy spaces that support no compact composition operators, J. Functional Anal. 205(2003), 62-89. MR2020208(2004h:30041)

Department of Mathematics and Statistics, American University of Sharjah, P. O. Box 26666, Sharjah, United Arab Emirates 